\title{
Shape Tolerance for Robot Gripper Jaws ${ }^{1}$
}

Tao Zhang, Lawrence Cheung and Ken Goldberg ${ }^{2}$

ALPHA Lab, IEOR and EECS Dept., UC Berkeley

\section{AbStraCt}

In [19] we specified robot grippers that can orient and grasp parts with an arrangement of trapezoidal jaw modules. Since jaw modules may be imprecisely machined, we define a parametric tolerance class such that part alignment is guaranteed for all jaw geometry in the class. This tolerance class is derived based on analysis of toppling, motion trajectory, and form-closure. Given maximal jaw geometry from the previous algorithm, we describe an $\mathrm{O}\left(\mathrm{n}^{3}\right)$ algorithm to compute the parametric tolerance class based on maximal and minimal jaw specifications. We have implemented the algorithm and report results from physical experiments.

\section{INTRODUCTION}

Although grippers are widely used for automated manufacturing, assembly, and packing, the design of gripper jaws is often ad-hoc and suboptimal. In industry, 4 DOF robots, such as SCARA arms, and 1 DOF parallel jaw grippers are common due to their low cost and high reliability. The combination of these two devices is kinematically limited to orienting parts in the horizontal plane. Zhang and Goldberg [19] gave an algorithm to design jaws based on trapezoidal modules that will align parts in the vertical plane and grasp them in form closure. The algorithm finds jaws that achieve maximal contact at the final grasp configuration to maximize resistance to applied forces.

For many industrial applications, it may be preferable to use jaws with smaller contact area, for example to minimize gripper weight for high-velocity transfer. Furthermore, machined jaws may not precisely comply with the specified maximal contact geometry. In this paper we consider variations in jaw shape and define a tolerance class for jaws based on maximal and minimal contact areas.

Let $\boldsymbol{J}_{\text {max }}$ denote the jaw specification from [19] that achieves maximal linear contacts with the part at its desired final orientation. Let $\boldsymbol{J}$ denote an instance of jaw geometry that is defined by an arrangement of trapezoidal jaw modules. We say $\boldsymbol{J}$ is admissible if it will rotate the part to the desired orientation and achieve form-closure. Let $\boldsymbol{J}_{\boldsymbol{m i n}}$ denote the admissible jaw geometry with minimal contacts at the desired orientation of the part. Figure 1 shows $\boldsymbol{J}_{\boldsymbol{m a x}}$ and $\boldsymbol{J}_{\text {min }}$ for a given part: both align the part to the desired orientation and achieve a form-closure grasp on the part. The tolerance class is bounded by $\boldsymbol{J}_{\max }$ and $\boldsymbol{J}_{\boldsymbol{m i n}}$.

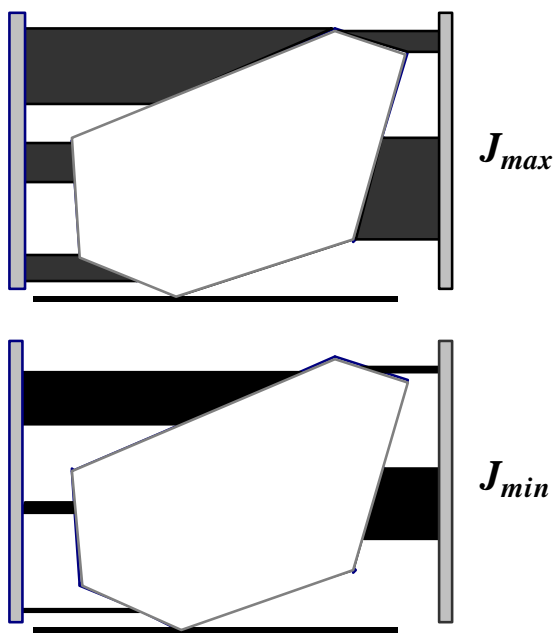

Figure 1 Black trapezoids illustrate the maximum and minimum boundaries of the jaw tolerance class for the part shown in gray.

As illustrated in Figure 2, the gripper with trapezoidal jaw modules rotates the part from its initial resting orientation (a) to the desired final orientation (b) for assembly. Our gripper design builds on recent results in toppling manipulation [8]. Zhang et al. [17] propose the toppling graph that can be used to identify the location of contacts permitting toppling. Zhang et al. [18] apply toppling to grasping and find four frictionless point contacts that will align a given part in the vertical plane. [19] gives an $O\left(n^{5}\right)$ algorithm to compute the maximal jaw design with linear contacts that has the following properties: (1) It is able to align the part from the initial orientation to the desired final orientation; (2) It has maximal (linear) contact with the part at the desired orientation of the part; and (3) It achieves a form-closure grasp on the part at its desired orientation.

In this paper, we develop a tolerance class specified as a range of trapezoidal jaws. Each jaw module is determined by the locations of two vertices that make contact with the part in its final grasp configuration. The line segment between these two vertices represents an accessible segment on an edge of the part at its desired orientation. The accessible segment corresponds to an edge of the jaw module that is neither horizontal nor vertical.

\footnotetext{
${ }^{1}$ This work was supported in part by the National Science Foundation under CDA-9726389 and Presidential Faculty Fellow Award IRI-9553197. Research funding was also provided by Adept Technology, Ford Motors, and California State MICRO Grant 00-032.

${ }^{2}$ For more information contact: goldberg@ieor.berkeley.edu.
} 


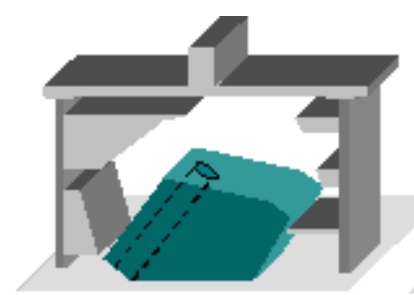

(a)

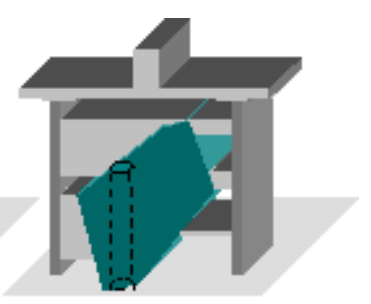

(b)
Figure 2 Gripper with trapezoidal jaw modules Dtates the part in the gravitational plane to facilitate assembly.

There are three types of vertices in the set of accessible segments: one pushing vertex, one toppling vertex, and other vertices named tips. As illustrated in Figure 3 , we define a single variational parameter $\lambda$ along the edge at each tip. As is common in tolerance analysis, we assume perfect form: all jaw modules in the tolerance class have perfect linear edges. We define the tolerance class by fixing the pushing and toppling vertices and computing how far the tips can be expanded or contracted along the accessible segments. Note that we define a single common tolerance parameter $\lambda$ for all tips.

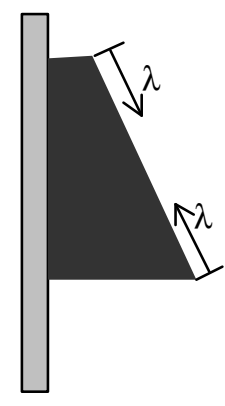

Figure 3 Variational parameters along an accessible segment of a jaw module.

We present an $O\left(n^{3}\right)$ algorithm for testing if a jaw specification $\boldsymbol{J}$ is admissible. Given $\boldsymbol{J}_{\text {max }}$, we then present an $O\left(n^{3}\right)$ algorithm to compute the tolerance class.

\section{RELATED WORK}

[9], [11], [12], and [15] survey the status quo and mathematical approaches to tolerancing. Neumann [10] describes a new standard, Y14.5M, which provides a mathematical basis for dimensioning and tolerancing.

A fundamental problem in geometric tolerancing is classification: given a part, is it within tolerance? Yap and Chang [16] give an example using a 1-dimensional probe model.

Configuration space can provide a theoretical basis for tolerance analysis. Donald [5] studies part manipulation with geometric uncertainty. He considers shape variations as an additional dimension in a gen- eralized configuration space and describes multi-step error detection and recovery strategies. Joskowicz et al. [6] present kinematic tolerance in term of configuration space and develop a worst-case tolerance analysis algorithm for 2-DOF planar pairs. Sack and Joskowicz [13] extend the analysis to multi-pair planar mechanisms with statistical geometric variation. They also model general planar part pairs using 3dimensional configuration-space to capture both quantitative and qualitative kinematic variation [14].

Latombe et al. [7] considers assembly sequence planning problem with toleranced parts. They give a polynomial time algorithm to decide if an assembly sequence exists given the specified tolerances. Their tolerance model is similar to ours in that both approaches fix the relative orientation of edges. Akella and Mason [1] develop a planner to generate orienting plans for toleranced polygonal parts. Their tolerance model is defined by circular uncertainty zones around the nominal positions of the COM and the vertices. Chen et al. [4] propose parameteric tolerance classes for sensorless part orienating and fixturing. Each are defined by a uncertainty zone at part vertices. They develop algorithms to compute the boundaries of the tolerance class. Brost and Peters [3] give an algorithm to design 3D modular fixtures for toleranced parts that are specified by an uncertainty polygon at each vertex. Bohringer et al. [2] show that toleranced parts can be oriented using an elliptic force field.

\section{PROBLEM DEFINITION}

Let I denote the input to the maximal jaw design algorithm in [19]: the $n$-sided convex projection of an extruded polygonal part, its COM, its initial and desired orientations, vertex clearance radius $\varepsilon, \mu_{\varphi}$ and $\mu_{s}$ : friction coefficients of gripper-part and surfacepart, respectively.

We first consider problem (1), testing if a given $\boldsymbol{J}$ is admissible (will rotate the part and hold it in form-closure). The input to problem (1) is $\langle\mathbf{I}, \boldsymbol{J}\rangle$. The output is binary: yes if $\boldsymbol{J}$ is admissible; no if not.

We then consider problem (2), finding the lower boundary of the tolerance class. The input of problem (2) is $\left\langle\mathbf{I}, \boldsymbol{J}_{\boldsymbol{m a x}}\right\rangle$. The output is $\boldsymbol{J}_{\boldsymbol{m i n}}$.

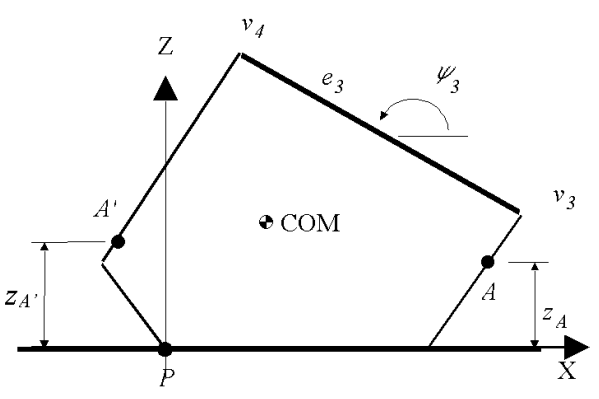

Figure 4 Notation. 
As shown in Figure 4, the part sits on a worksurface at an initial resting pose. We define the World frame, $\boldsymbol{W}$, to be a Cartesian coordinate system originating at pivot point $P$ with $\mathrm{X}$-axis on the surface pointing right, Z-axis vertical to the surface pointing up. The pushing contact, $A^{\prime}$, is a distance $z_{A}$, from the surface; the toppling contact, $A$, is a distance $z_{A}$ from the surface. Starting from the pivot, we consider each edge of the part in counter-clockwise order, namely $e_{1}, e_{2}, \ldots, e_{n}$. The edge $e_{i}$, with vertices $v_{i}$ at $\left(x_{i}, z_{i}\right)$ and $v_{(i+1)}$ at $\left.\left(x_{(i+1)}\right), z_{(i+1)}\right)$, is in direction $\psi_{i}$ from the $\mathrm{X}$ axis.

Let $\boldsymbol{\theta}$ denote the rotation angle of the part from the $+\mathrm{X}$ direction; initially $\boldsymbol{\theta}=0$ and at the final orientation $\boldsymbol{\theta}=\boldsymbol{\theta}_{d}$. We say an edge $e_{k}$ is visible if it can be seen from $+\mathrm{X}$ direction; invisible, otherwise.

We assume the part can be treated as a rigid extrusion of a polygon; both the part and the jaws are rigid; part geometry and location of the COM are known; part motion is sufficiently slow to apply quasi-static analysis.

\section{TOLERANCE ANALYSIS}

The tolerance analysis is a combination of toppling, motion trajectory, and form-closure study.

\subsection{Toppling graph}

Our analysis involves the graphical construction of a set of shape functions that represent the mechanics of grasping. All of these functions are piecewise sinusoidal and dependent on $\boldsymbol{\theta}$. They map from part orientation to height: $S^{1} \rightarrow \boldsymbol{\Re}^{+}$, where $S^{1}$ is the set of planar orientations. The shape functions include vertex functions $V_{j}(\boldsymbol{\theta})$, toppling functions $H_{j}(\boldsymbol{\theta})$, and jamming functions $J_{j}(\boldsymbol{\theta})$.

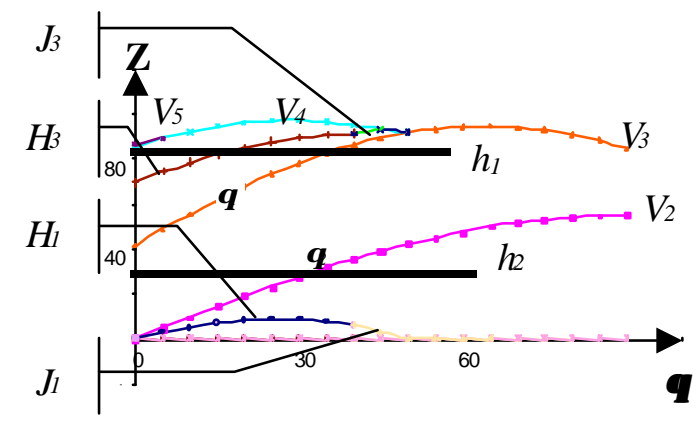

Figure 5 Toppling Graph.

The toppling graph, which consists of these shape functions, helps us to identify the range of the contact permits toppling. Each function represents a particular property of the part, and the graph describes properties of the part during grasping.
For toppling to be successful, there must exist a horizontal line at height $h$ that has the following characteristics:

$\begin{array}{ll}\text { 1. } h>H_{i}(\boldsymbol{\theta}), \text { if } V_{i}(\boldsymbol{\theta})<h<V_{i+1}(\boldsymbol{\theta}) ; & \# \mathbf{1} \\ \text { 2. } h>J_{i}(\boldsymbol{\theta}), \text { if } V_{i}(\boldsymbol{\theta})<h<V_{i+1}(\boldsymbol{\theta}) ; & \# \mathbf{2} \\ \text { 3. } h<\max _{i}\left(V_{i}(\boldsymbol{\theta})\right) \text {, where } 0<\boldsymbol{\theta}<\boldsymbol{\theta}_{d} . & \mathbf{\# 3}\end{array}$

where $i$ is the index of visible edges.

The first three criteria can be described as: the toppling contact $A$ must be above the toppling function, the jamming function, and the liftoff function. When the horizontal line crosses a vertex function, there is a contact edge switch. Therefore, $A$ must satisfied criteria 1 and 2 for the new contact edge. The third criterion requires that $A$ must make contact with the part.

For example, we want to rotate a sample part $35^{\circ}$ for assembly. Figure 5 illustrates the toppling graph of the part given $z_{A}{ }^{\prime}=0.5 \mathrm{~cm}$. Note that $H_{2}$ and $J_{2}$ equal 0 . We can see that $A$ at $z_{A}=h_{l}$ is unable to topple the part to the desired orientation because the line goes under $H_{3}$ after rotating to $\Theta_{l} ; A$ at $z_{A}=h_{2}$ is capable to perform the task. Notice that $A$ switches contact edge from $e_{2}$ to $e_{1}$ at $\boldsymbol{\theta}_{2}$.

Given $z_{A}$, the toppling graph allows us to find the feasible range of $z_{A}$ such that the corresponding $A$ and $A^{\prime}$ can rotate the part from the resting orientation to the desired orientation.

\subsection{Trajectory analysis}

To ensure no portion of the jaw blocks the part rotation, we define quasi-vertex functions to represent the motion trajectory of vertices.

The part performs both rotation and linear translation during toppling. We decompose the part motion into pure rotation and pure translation. The part first rotates about pivot point $P$ to semi-position, and then translates to actual-position. Let $\left({ }_{\theta} x_{j},{ }_{\theta} z_{j}\right)$ and $\left({ }_{\theta}\right.$ $\left.x_{j}^{\prime}, \theta \quad z_{j}^{\prime}\right)$ denote the actual-position and the semiposition of vertex $v_{j}$ after the part is toppled by $\boldsymbol{\theta}$, respectively. Let $\left({ }_{d} x_{j},{ }_{d} z_{j}\right)$ and $\left({ }_{d} x_{j}{ }_{j},{ }_{d} z_{j}{ }_{j}\right)$ denote the actual-position and the semi-position of vertex $v_{j}$ after the part is toppled to its desired orientation, respectively. Let ${ }_{\theta} x_{t}$ and ${ }_{d} x_{t}$ denote the distance between the actual-position and the semi-position of any point after the part is toppled by $\boldsymbol{\theta}$ and $\boldsymbol{\theta}_{d}$, respectively. To obtain a quasi-vertex function, we define a frame of reference $F_{j}$ at the desired orientation of the part originating at $v_{j}$. The Z-axis of $F_{j}$ is the interior normal of edge $e_{(j-1)}$, and the X-axis is on edge $e_{(j-1)}$ obeying the right-hand rule.

Given $z_{A}$, the quasi-vertex function $Q_{j k}\left(\theta, z_{A}\right)$ indicates the location of $v_{k}$ in $F_{j}$ as the part rotates, which can be shown to be:

$Q_{j k}(\boldsymbol{\theta})=\left[\begin{array}{l}{ }_{x} Q_{j k}\left(\boldsymbol{\Theta}, z_{A}\right) \\ { }_{z} Q_{j k}\left(\boldsymbol{\Theta}, z_{A}\right)\end{array}\right]$ 
$\left[\begin{array}{l}\left({ }_{\theta} x_{k}^{\prime}-{ }_{d} x_{j}^{\prime}{ }_{\theta} x_{t}-{ }_{d} x_{t}\right) \cos \left(\boldsymbol{\Psi}_{j-1}+\boldsymbol{\Theta}_{d}\right)+\left({ }_{\theta} z_{k}^{\prime}{ }_{d} z_{j}^{\prime}\right) \sin \left(\boldsymbol{\Psi}_{j-1}+\boldsymbol{\Theta}_{d}\right) \\ -\left({ }_{\theta} x_{k}^{\prime}{ }_{k}-{ }_{d}^{\prime}{ }_{j}{ }_{j}{ }_{\theta} x_{t}-{ }_{d} x_{t}\right) \sin \left(\boldsymbol{\Psi}_{j-1}+\boldsymbol{\Theta}_{d}\right)+\left({ }_{\theta} z_{k}^{\prime}{ }_{d} z_{j}^{\prime}{ }_{j}\right) \cos \left(\boldsymbol{\Psi}_{j-1}+\boldsymbol{\Theta}_{d}\right)\end{array}\right]$

\# 4

where $\left[\begin{array}{c}{ }_{d} x_{j}^{\prime} \\ 0 \\ { }_{d} z_{j}^{\prime} \\ 1\end{array}\right]=\left[\begin{array}{c}x_{j} \cos \theta_{d}-z_{j} \sin \theta_{d} \\ 0 \\ x_{j} \sin \theta_{d}+z_{j} \cos \theta_{d} \\ 1\end{array}\right]$

${ }_{d} x_{t}=x_{A}-\frac{\left(z_{A}-{ }_{d} z_{m}^{\prime}\right)\left({ }_{d} x_{m+1}^{\prime}-{ }_{d} x_{m}^{\prime}\right)}{{ }_{d} z_{m+1}^{\prime}-{ }_{d} z_{m}^{\prime}}-{ }_{d} x_{m}^{\prime}$ if ${ }_{d} z_{m}<$

$z_{A}<_{d} z_{m+1}^{\prime}$,

and ${ }_{\theta} x_{t}=x_{A}-\frac{\left(z_{A}-{ }_{\theta} z_{l}^{\prime}\right)\left({ }_{\theta} x_{l+1}^{\prime}-{ }_{\theta} x_{l}^{\prime}\right)}{{ }_{\theta} z^{\prime}{ }_{l+1}-{ }_{\theta} z_{l_{l}}}-{ }_{\theta} x_{l}^{\prime}$ if ${ }_{\theta} z_{l}{ }_{l}<$ $z_{A}<{ }_{\theta} z^{\prime} l+1$

We represent the motion trajectory of the edges of the part based upon the quasi-vertex functions, and then we derive the accessible segments of the jaws. To guarantee no obstacle blocks the part rotation, the jaws should stay away from the motion trajectory of the edges.

The quasi-vertex function describes the motion trajectory of the part. Note that the quasi-vertex function is the projection of configuration-space $(x, z, \theta)$ onto the plane of $(x, z)$; the shape function is the project of configuration-space $(x, z, \theta)$ onto the plane of $(\theta, z)$. Therefore, the shape function and the quasi vertex function are both the decomposition of the configuration-space. The reason we apply $C$-space decomposition is that, in order to reduce complexity, we only need to keep a portion of the configurationspace information that is necessary for certain analysis.

\subsection{Problem (1): Checking if $\boldsymbol{J}$ is admissible}

To solve problem (1) - checking if a given $\boldsymbol{J}$ is admissible, we first test if $\boldsymbol{J}$ will achieve form-closure on the part.

Starting from the one closest to $P$, we order the vertices of $\boldsymbol{J}$ in counter-clockwise $\left(a_{1}, b_{1}\right),\left(a_{2}, b_{2}\right)$, $\ldots,\left(a_{m}, b_{m}\right)$. Let $V_{k}$ denote the unit normal vector pointing inward at $\left(a_{k}, b_{k}\right), V_{k a}\left(V_{k b}\right)$ denote the $\mathrm{X}(\mathrm{Z})$ axis projection of this unit vector, and $T_{k}$ denote the torque of $V_{k}$ respective to $P$.

Let $\mathbf{M}$ denote $\left[\begin{array}{ccccc}V_{1 a} & V_{2 a} & V_{3 a} & \ldots & V_{m a} \\ V_{1 b} & V_{2 b} & V_{3 b} & \ldots & V_{m b} \\ T_{1} & T_{2} & T_{3} & \ldots & T_{m}\end{array}\right]$, let $\boldsymbol{\omega}$ denote $\left[\omega_{1} \omega_{2} \omega_{3} \ldots \omega_{m}\right]^{T}$.

It is well known that $\boldsymbol{J}$ generates a form-closure grasp on the part if and only if $\exists \boldsymbol{\omega}>0$, s.t. $\mathbf{M} \boldsymbol{\omega}=0$. Therefore, to check the form-closure grasps is equivalent to solve a system of equations and can be done in $O\left(n^{3}\right)$.

Second, we need to test if $\boldsymbol{J}$ is able to rotate the part to the desired final orientation.

Since the part is rolled by the pushing contact and the toppling contact, we need to identify these two points. These two contacts are only vertices that keep touch with the part during the toppling phase. This can be done easily in time $O(n)$.

Known the height of the pushing contact $z_{A}$, we construct the corresponding toppling graph. If $h=z_{A}$ satisfies inequality \#1 \#3, the pair of $A$ and $A$ ' can rotate the part to the final orientation. Since the time to obtain a toppling graph is $O(n)$, this step takes $O(n)$.

Finally, we need to consider if any portion of $\boldsymbol{J}$ will block the part's trajectory.

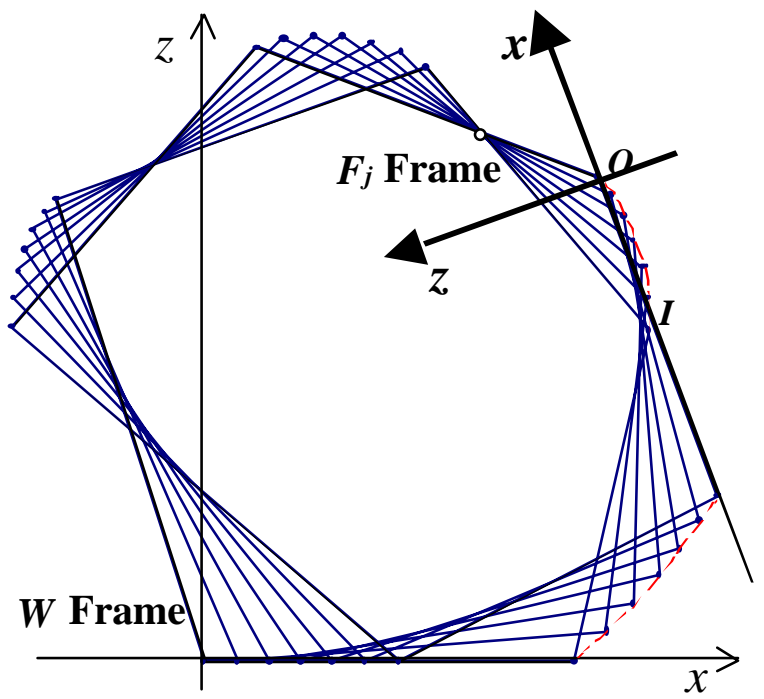

Figure 6 Motion trajectory of the part.

From equation \#4, we have:

${ }_{x} Q_{j k}{ }^{2}+{ }_{z} Q_{j k}{ }^{2}=\left({ }_{\theta} x_{k}^{\prime}-{ }_{d} x_{j}^{\prime}+{ }_{\theta} x_{t}-{ }_{d} x_{t}\right)^{2}+\left({ }_{\theta} z_{k}^{\prime}-{ }_{d} z_{j}^{\prime}\right)^{2}$.

Therefore, the intersection between $Q_{j k}(\boldsymbol{\theta})$ and the $\mathrm{X}$ axis of $\boldsymbol{F}_{j}$ is at:

$x_{j k}=\sqrt{\left(\left({ }_{d} x_{j}^{\prime}{ }_{j} x_{t}\right)-\left({ }_{\theta} x_{k}^{\prime}+{ }_{\theta} x_{t}\right)\right)^{2}+\left({ }_{\theta} z_{k}^{\prime}-{ }_{d} z_{j}^{\prime}\right)^{2}}$

where $\tilde{\boldsymbol{\theta}}$ satisfies:

${ }_{z} Q_{j k}\left(\tilde{\boldsymbol{\theta}}, z_{A}\right)=$

$-\left({ }_{\tilde{\theta}} x_{k}^{\prime}-{ }_{d} x_{j}^{\prime}+{ }_{\tilde{\theta}} x_{t}-{ }_{d} x_{t}\right) \sin \left(\boldsymbol{\Psi}_{j-1}+\boldsymbol{\Theta}_{d}\right)+\left({ }_{\tilde{\theta}} z_{k}^{\prime}-{ }_{d} z^{\prime}{ }_{j}\right) \cos \left(\boldsymbol{\Psi}_{j-1}+\boldsymbol{\Theta}_{d}\right)$

$=0$.

Figure 6 illustrates the motion of the part. $O$ is the origin of $\boldsymbol{F}_{j}$ at $v_{j}$, and $I$ is the intersection between 
the quasi-vertex function and the X-axis of frames $\boldsymbol{F}_{\boldsymbol{j}}$. Therefore, $x_{j k}$ is the length of $\overline{O I}$. We project $\overline{O I}$ to the $\mathrm{X}$-axis of $\boldsymbol{W}$, and the length of the resulting segment is $\left({ }_{d} x_{j}^{\prime}+{ }_{d} x_{t}\right)-\left(\underset{\theta}{ } x_{k}^{\prime}{ }_{i} x_{\theta}\right)$; we project $\overline{O I}$ to the Zaxis of $\boldsymbol{W}$, and the length of the resulting segment is $\tilde{\theta}_{\tilde{\theta}} z_{k}^{\prime}-{ }_{d} z^{\prime}{ }_{j}$. If $x_{j k}$ of $\boldsymbol{J}$ is smaller than that of $\boldsymbol{J}_{\max }$ (where $j$ and $k$ correspond to all the visible edges), no portion of the jaws will become an obstacle in the trajectory. We compute $x_{j k}$ of $\boldsymbol{J}$ and that of $J_{\text {max }}$, and compare these two values for all $j$ and $k$. Thus the algorithm to solve Problem (1) runs in time $O\left(n^{3}\right)$.

\subsection{Problem (2): Computing the tolerance class}

$\left[\boldsymbol{J}_{\min }, \boldsymbol{J}_{\max }\right]$ defines a tolerance class: the uncountable set of grippers with jaws having edges parallel to $\boldsymbol{J}_{\boldsymbol{m i n}}$ and $\boldsymbol{J}_{\boldsymbol{m a x}}$ and volume within these lower and upper boundaries. We first prove the convexity of the tolerance class: if $\boldsymbol{J} \in\left[\boldsymbol{J}_{\min }, \boldsymbol{J}_{\max }\right], \boldsymbol{J}$ must be admissible. Then we describe an algorithm to find $J_{\min }$ by searching the upper bond of $\lambda$.

Lemma 1. If $\boldsymbol{J} \in\left[\boldsymbol{J}_{\min }, \boldsymbol{J}_{\max }\right], \boldsymbol{J}$ must be admissible. Proof: Note that both $\boldsymbol{J}_{\min }$ and $\boldsymbol{J}_{\max }$ are admissible by definition.

(1) Can $\boldsymbol{J}$ topple the part to desired orientation?

First, we consider the toppling conditions. Since the pushing contact and the toppling contact are the same for all $\boldsymbol{J}$ in the class, the toppling condition of $\boldsymbol{J}$ is the same as that of $\boldsymbol{J}_{\text {max }}$. Second, we consider the part's motion trajectory conditions. The smaller $\boldsymbol{J}$, the less likely it will block the motion trajectory of the part. Since $\boldsymbol{J}_{\max }$ is the geometry shape that guarantees no collision in the part's motion trajectory and $\boldsymbol{J}$ is smaller than $J_{\text {max }}, J$ satisfies the motion trajectory conditions. Therefore, $\boldsymbol{J}$ is able to topple the part to desired orientation.

(2) Can $\boldsymbol{J}$ achieve a form-closure grasp on the part at its desired orientation?

$\boldsymbol{J}$ is larger than $\boldsymbol{J}_{\text {min }}$. Since $\boldsymbol{J}_{\text {min }}$ achieve a formclosure grasp on the part at its desired orientation, $\boldsymbol{J}$ must have the same property.

In summary, $\boldsymbol{J}$ can topple the part to desired orientation and achieve a form-closure grasp on the part at its desired orientation. Therefore, $\boldsymbol{J}$ is admissible.

Lemma 2. $\lambda$ must be nonnegative for $\boldsymbol{J}$.

Proof: Assume that there exists a $\lambda$ that is negative for certain jaw geometry $\boldsymbol{J}$. Then, the total length of the contact edges of $\boldsymbol{J}$ are longer than that of $\boldsymbol{J}_{\text {max }}$ because $\lambda<0$. Since $\boldsymbol{J}_{\max }$ has maximal contacts with the part at the desired orientation, some portion of $\boldsymbol{J}$ will become an obstacle in the part rotation trajectory. Therefore, $\lambda$ can only be nonnegative.

Numerical Algorithm: We use binary search to find the maximum variational parameter $\lambda$. By Lemma 2 $\lambda$ must be nonnegative. We choose a small positive number $\delta$. Starting with $\lambda=\delta$, we use the algorithm for Problem (1) to check if the corresponding $J$ is admissible. If so, we try $\lambda=2 \delta$, and so on, until $\lambda$ is sufficiently large that $\boldsymbol{J}$ is not admissible. We then interpolate to a desired level of accuracy and the corresponding $\boldsymbol{J}$ is $\boldsymbol{J}_{\boldsymbol{m i n}}$.

\section{IMPLEMENTATION RESULTS}

We verify our shape tolerance algorithms by the following example. The part is initially at the stable orientation defined by the vertices at $(0,0),(51.2,0)$, (64.1, 57.2), (37.5, 96.2), (-32.2, 44.6), and COM at $(21.9,42.3)$. We need to rotate the part $20^{\circ}$ to final orientation for assembly.

\begin{tabular}{|c|c|c|c|}
\hline$a_{1}$ & 46.41 & $b_{1}$ & 21.64 \\
\hline$a_{2}$ & 33.94 & $b_{2}$ & 78.97 \\
\hline$a_{3}$ & 33.94 & $b_{3}$ & 78.97 \\
\hline$a_{4}$ & 5.93 & $b_{4}$ & 95.61 \\
\hline$a_{5}$ & -6.65 & $b_{5}$ & 103.08 \\
\hline$a_{6}$ & -39.76 & $b_{6}$ & 42.04 \\
\hline$a_{7}$ & -48.04 & $b_{7}$ & 26.78 \\
\hline$a_{8}$ & -6.17 & $b_{8}$ & 4.55 \\
\hline
\end{tabular}

Table 1 Optimal jaw design: vertex location.

We find the optimal gripper jaw design as shown in Figure 7. Table1 indicates the location of the jaw module vertices. The toppling contact is at $\left(a_{2}, b_{2}\right)$ and the pushing contact is at $\left(a_{6}, b_{6}\right)$. We apply our algorithm to find the upper bond of $\lambda$ equals 22 as illustrated in Figure 8.

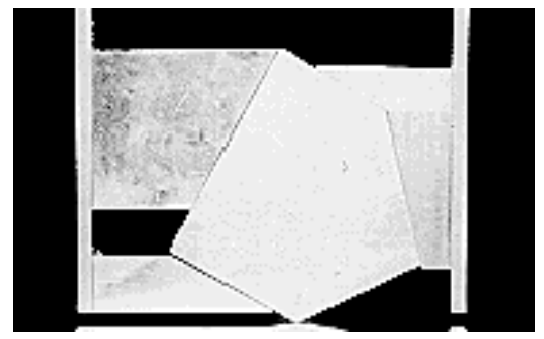

Figure $7 \boldsymbol{J}_{\max }$ rotates the part and grasps it securely at the desired orientation.

We conducted physical experiments to verify our results on the example part. Two sets of jaws were machined from aluminum. The friction coefficients are $\mu_{q}=0.0875$ and $\mu_{s}=0.0875$. The first set is $\boldsymbol{J}_{\max }$ as shown in Figure 7. The second set is $\boldsymbol{J}_{\min }$ as shown in Figure 8. We installed these two sets of the jaws onto an AdeptOne industrial robot. We tested each $\boldsymbol{J}_{\text {max }}$ and $\boldsymbol{J}_{\text {min }} 50$ times to align the part and observed zero failures. Figure 7 and 8 illustrate, in sequence, 
both jaw sets successfully rotate the part to the desired orientation and grasp it securely.

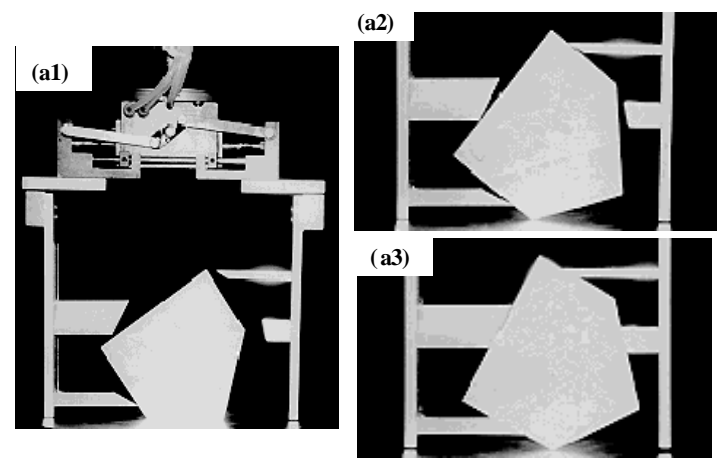

Figure 8 Physical experiments for $\boldsymbol{J}_{\min }$.

\section{DISCUSSION AND FUTURE WORK}

It is very difficult to characterize the grasping proprieties of the jaws with uncountable shape uncertainty. Therefore, we intend to quickly check the orientability of jaws during interactive design cycle. Algorithms with low complexity, such as those described in this paper, can provide rapid feedback to designers.

We propose a rigorous parametric tolerance class to address the shape uncertainty of the gripper jaws. We study shape tolerance of the jaws in terms of toppling graph, part's motion trajectory, and formclosure. We present a fast checking algorithm, and use it to compute the tolerance class. We implement the algorithms and illustrate with physical examples.

In the future, we will study sensitivity to changes in friction coefficient and consider alternative materials for gripper jaws. We will also consider sensitivity in jaw shape normal to the contacting surfaces, which may justify use of deformable materials such as rubber for the contacting surfaces. The idea is to design jaws that are also robust to variations in part shape.

\section{REFERENCES}

[1] S. Akella and M. Mason. "Orienting toleranced polygonal parts," Int. J. Robot. Res., vol. 19, no. 12, pp. 1147-1170, 2000.

[2] K. Bohringer, B. Donald, L. Kavraki, and F. Lamiraux. "Part orientation with one or two stable equilibria using programmable force fields," IEEE Trans. Robot. Automat., vol. 16, no. 2, pp. 157-170, 2000.

[3] R. Brost and R. Peters. "Automatic design of 3-D fixtures and assembly pallets," Int. J. Robot. Res., vol. 17, no. 12, pp. 1243-1281, 1998.

[4] J. Chen, K. Goldberg, M. Overmas, D. Halperin, K. Bohringer, and Y. Zhuang. "Shape tolerance in feeding and fixturing," in $R o$ - in Robotics: The Algorithmic Perspective, ed. P. Agarwal, L. Kavraki, and M. Mason. A. K. Peters, 1999.

B. Donald. "Planning multi-step error detection and recovery strategies," Int. J. Robot. Res., vol. 9, no. 1, pp. 3-60, 1990.

[6] L. Joskowicz, E. Sacks, and V. Srinivasan. "Kinematic tolerance analysis," ComputerAided Design, Vol. 29, No. 2, pp. 147-157, 1996.

[7] J.-C. Latombe, R. Wilson, and F. Cazals. "Assembly sequencing with toleranced parts," Computer-Aided Design, Vol. 29, No. 2, pp. 159-174, 1997.

[8] K. Lynch. "Toppling manipulation," in IEEE Int. Con. Robot. Automat., Detroit, 1999, pp. 2551-2557.

[9] J. Meadows. Geometric Dimensioning and Tolerancing, Marcel Dekker, Inc., 1995.

[10] A. Neumann. "The new Y14.5M sandard on dimensioning and tolerancing," Manufacturing Review, Vol. 7, No. 1, pp. 9-15, 1994.

[11] A. Requicha. "Mathematical definition of tolerance specifications," Manufacturing Review, Vol. 6, No. 4, pp. 269-274, 1993.

[12] U. Roy, C. Liu, and T. Woo. "Review of dimensioning and tolerancing: representation and processing," Computer-Aided Design, Vol. 23, No. 7, pp. 466-483, 1991.

[13] E. Sacks and L. Joskowicz. "Parametric knematic tolerance analysis of planar mechanisms," Computer-Aided Design, Vol. 29, No. 5, pp. 333-341, 1997.

[14] E. Sacks and L. Joskowicz. "Parametric knematic tolerance analysis of general planar systems," Computer-Aided Design, Vol. 30, No. 9, pp. 707-714, 1998.

[15] H. Voelcker. "A current perspective on tolerancing and metrology," Manufacturing Review, Vol. 6, No. 4, pp. 258-268, 1993.

[16] C. Yap and E. Chang. "Issues in the metrology of geometric tolerancing," in J.-P. Laumond and M. Overmas, editors, Algorithms for Robotic Motion and Manipulation, pp. 393-400, A. K. Peters, 1997.

[17] T. Zhang, G. Smith, R. Berretty, M. Overmars, and K. Goldberg. "The toppling graph: designing pin sequences for part feeding," in Proc. IEEE Int. Conf. Robot. Automat., San Francisco, 2000, pp. 139-146.

[18] T. Zhang, G. Smith and K. Goldberg. "Compensatory grasping with the paralleljaw gripper," in $4^{\text {th }}$ Int. Workshop Algorithmic Foundations Robot., Hanover, NH, 2000.

[19] T. Zhang and K. Goldberg. "Design of gripper jaws based on trapezoidal modules," in Proc. IEEE Int. Conf. Robot. Automat., Seoul, Korea, 2001. 